\title{
Kubo-Greenwood approach to conductivity in dense plasmas with average atom models
}

\author{
C. E. Starrett ${ }^{1, *}$ \\ ${ }^{1}$ Los Alamos National Laboratory, P.O. Box 1663, Los Alamos, NM 87545, U.S.A.
}

(Dated: April 8, 2016)

\begin{abstract}
A new formulation of the Kubo-Greenwood conductivity for average atom models is given. The new formulation improves upon previous treatments by explicitly including the ionic-structure factor. Calculations based on this new expression lead to much improved agreement with ab initio results for DC conductivity of warm dense hydrogen and beryllium, and for thermal conductivity of hydrogen. We also give and test a slightly modified Ziman-Evans formula for the resistivity that includes a non-free electron density of states, thus removing an ambiguity in the original Ziman-Evans formula. Again results based on this expression are in good agreement with ab initio simulations for warm dense beryllium and hydrogen. However, for both these expressions, calculations of the electrical conductivity of warm dense aluminum lead to poor agreement at low temperatures compared to $a b$ initio simulations.
\end{abstract}

Keywords: electron conductivity, warm dense matter, average atom model, Ziman formula, Kubo-Greenwood

\section{INTRODUCTION}

An important aspect of modeling warm and hot dense matter is the calculation of electron thermal and electrical conductivities. The former is of particular relevance in the field of inertial confinement fusion [1, 2] where it is the main phenomenon that determines the ablation of the cold deuterium/tritium fuel. Currently we have no reliable model that can predict accurate thermal and electrical conductivities across all temperature and density regimes of interest. In particular, as we move out of the degenerate electron regime the reliable method of Kohn-Sham density functional theory molecular dynamics (KS-DFT-MD) coupled with the Kubo-Greenwood formalism [3-6] quickly becomes computationally prohibitive. In the degenerate, or nearly degenerate regimes, this method is thought to be accurate and agrees with experiments for materials under normal conditions [7].

Average atom models provide a computationally efficient alternative at the cost of physical accuracy. The central idea is that one tries to calculate the properties of one atom in the plasma that is supposed to represent the average of all atoms in the plasma. Average atom models have been used successfully for many years for equation of state calculations [8-13]. They have also been used for electrical conductivity calculations, primarily by coupling to the Ziman-Evans (ZE) formula [1420]. Recently, a systematic comparison of calculations of electrical conductivity using this method against KuboGreenwood KS-DFT-MD calculations [14] showed generally very good agreement between the methods provided that a judicious choice was made when coupling the average atom model to the $\mathrm{ZE}$ formula. However, the $\mathrm{ZE}$ formula, unlike the KG method, is not easily generalized to thermal conductivity or optical conductivity. The lat-

*Electronic address: starrett@lanl.gov ter is useful as it can by used to calculate other optical properties, including the opacity and reflectivity [21].

A formulation of the Kubo-Greenwood method for average atoms models has been developed by Johnson and co-workers [22-24]. However, a subsequent systematic analysis of the method compared to KS-DFT-MD showed some serious inaccuracies [25]. Unlike the ZE formulation, Johnson's KG formulation does make not explicit account of the ion-ion structure factor $S(k)$. In this work, we give an alternative derivation of the KG formulation for average atom models that explicitly accounts for $S(k)$. The new formulation recovers Johnson's result when $S(k)=1$. We also give the equations for thermal and optical conductivity.

To evaluate this new formulation we make comparisons to KS-DFT-MD calculations for hydrogen [1] and beryllium [5]. We also compare to other models [7, 15] and experiments for aluminum [26, 27]. We use the recently developed pseudo-atom molecular dynamics (PAMD) $[28,29]$ to generate the necessary inputs for the KG equation.

In addition to this, we present a slightly modified Ziman-Evans formula that takes into account a non-free electron density of states (DOS). The original ZE formula assumes a free electron DOS and this leads to an ambiguity in the choice of chemical potential and density of scattering electrons. This point was discussed in detail in [14]. The present reformulation recovers the original form of the $\mathrm{ZE}$ equation when the DOS goes to the free electron form and removes the ambiguity when the DOS is not free electron like. We compare calculations based on this new $\mathrm{ZE}$ formulation to the new $\mathrm{KG}$ formulation and to the KS-DFT-MD results.

The structure of this paper is as follows. In section II we derive the Kubo-Greenwood expression for average atom models with explicit account of the ion-ion structure factor. We also give the expression for the thermal conductivity. In section III we show how the ZimanEvans formula for the inverse resistivity is modified to 
account for a non-free electron density of states. In section IV we discuss the connection of these formulas to the the Pseudo-Atom Molecular Dynamics (PAMD) average atom model. In section $\mathrm{V}$ we use the PAMD model with the new KG and ZE expressions to calculate the DC electrical conductivity of warm dense hydrogen, beryllium and aluminum, and compare to available simulations, models and experiments. For hydrogen we also compare thermal conductivity calculations to KS-DFTMD simulation results. Lastly, in section VI we draw our conclusions. Throughout we use Hartree atomic units in which $\hbar=m_{e}=e=1$.

\section{KUBO-GREENWOOD APPROXIMATION}

The Kubo-Greenwood expression for the conductivity is $[4]$

$$
\begin{aligned}
\sigma(\omega)= & \frac{-2 \pi}{V} \int d \epsilon \frac{f\left(\epsilon_{m}\right)-f\left(\epsilon_{n}\right)}{\omega} \int d^{3} k_{m} \int d^{3} k_{n} \\
& \times\left\langle\left|J_{m n}\right|^{2}\right\rangle \delta\left(\epsilon_{m}-\epsilon-\omega\right) \delta\left(\epsilon_{n}-\epsilon\right)
\end{aligned}
$$

with

$$
J_{m n} \equiv \int_{V} d^{3} r \psi_{\boldsymbol{k}_{m}}^{*}(\boldsymbol{r}) \hat{\boldsymbol{v}}_{z} \psi_{\boldsymbol{k}_{n}}(\boldsymbol{r})
$$

where $\epsilon_{n(m)}=k_{n(m)}^{2} / 2$ is the energy of the initial (final) electron state and $\psi_{\boldsymbol{k}_{n(m)}}(\boldsymbol{r})$ is the corresponding wave function, $f(\epsilon)$ is the Fermi-Dirac occupation factor and $\hat{\boldsymbol{v}}_{z}$ is the velocity operator in the $\hat{\boldsymbol{z}}$ direction. Following Evans [30], we now assume that the potential felt by a electron is of muffin-tin form. In this widely used approximation the total scattering potential is the sum of non-overlapping potentials, centered on each nuclear site. Each muffin-tin potential is contained in a sphere of volume $V_{M T}$. Again following Evans [30] we further assume that the wave function inside each sphere is given by

$$
\psi_{\boldsymbol{k}_{n}}(\boldsymbol{r})=\bar{\psi}_{\boldsymbol{k}_{n}}(\boldsymbol{r}) e^{\imath \boldsymbol{k}_{n} \cdot \boldsymbol{R}_{\alpha}}
$$

where

$$
\bar{\psi}_{\boldsymbol{k}_{n}}(\boldsymbol{r})=\sum_{l} \sum_{m} \imath^{l} e^{\imath \delta_{l}\left(k_{n}\right)} Y_{l m}\left(\hat{\boldsymbol{k}_{n}}\right) Y_{l m}^{*}\left(\hat{\boldsymbol{r}_{n}}\right) \frac{y_{l}\left(r, k_{n}\right)}{r \sqrt{k}}
$$

Here $\boldsymbol{R}_{\alpha}$ is the position vector of nucleus $\alpha$. Further assuming that each muffin tin potential is identical and using the definition of the ion-ion structure factor:

$$
S(k)=\frac{1}{N}\left\langle\rho_{\boldsymbol{k}} \rho_{-\boldsymbol{k}}\right\rangle
$$

where

$$
\rho_{\boldsymbol{k}}=\sum_{\alpha=1}^{N} e^{\imath \boldsymbol{k} \cdot \boldsymbol{R}_{\alpha}}
$$

the Kubo-Greenwood conductivity expression is reduced to

$$
\begin{aligned}
\sigma(\omega)= & -2 \pi n_{I}^{0} \int d \epsilon \frac{f\left(\epsilon_{m}\right)-f\left(\epsilon_{m}\right)}{\omega} \int d \hat{\boldsymbol{k}}_{m} \int d \hat{\boldsymbol{k}}_{n} \\
& \times\left|\sqrt{k_{n} k_{m}} \bar{J}_{m n}\right|^{2} S\left(\left|\boldsymbol{k}_{n}-\boldsymbol{k}_{m}\right|\right)
\end{aligned}
$$

where $\epsilon_{m}=\epsilon+\omega, \epsilon_{n}=\epsilon, n_{I}^{0}=N / V$

$$
\bar{J}_{m n} \equiv \int_{V_{M T}} d^{3} r \bar{\psi}_{\boldsymbol{k}_{m}}^{*}(\boldsymbol{r}) \hat{\boldsymbol{v}}_{z} \bar{\psi}_{\boldsymbol{k}_{n}}(\boldsymbol{r})
$$

Using equation (4) in (7) and after some lengthy algebra (see appendix) we arrive at the result

$$
\sigma(\omega)=\sigma^{(1)}(\omega)+\sigma^{(2)}(\omega)+\sigma^{(3)}(\omega)
$$

with

$$
\begin{aligned}
& \sigma^{(1)}(\omega)=-2 \pi n_{I}^{0} \int d \epsilon \frac{f\left(\epsilon_{m}\right)-f\left(\epsilon_{m}\right)}{\omega} \\
& \times \sum_{l=0}^{\infty}\left|I_{A}(l)\right|^{2} \frac{(l+1)}{2(2 l+3)}\left[\left(1+\frac{l}{2}\right) I_{s}^{(1)}+\frac{l}{2} I_{s}^{(2)}\right] \\
& \sigma^{(2)}(\omega)=2 \pi n_{I}^{0} \int d \epsilon \frac{f\left(\epsilon_{m}\right)-f\left(\epsilon_{m}\right)}{\omega} \\
& \times \sum_{l=0}^{\infty} I_{A}(l) I_{B}(l+2) \cos \left(\delta_{l}\left(k_{n}\right)-\delta_{l+2}\left(k_{n}\right)\right) \\
& \times \frac{(l+1)(l+2)}{2(2 l+3)}\left[3 I_{s}^{(2)}-I_{s}^{(1)}\right] \\
& \sigma^{(3)}(\omega)=-2 \pi n_{I}^{0} \int d \epsilon \frac{f\left(\epsilon_{m}\right)-f\left(\epsilon_{m}\right)}{\omega} \\
& \times \sum_{l=0}^{\infty}\left|I_{B}(l)\right|^{2} \frac{l}{4(2 l-1)}\left[(l-1) I_{s}^{(1)}+(l+1) I_{s}^{(2)}\right]
\end{aligned}
$$

where

$$
\begin{gathered}
I_{s}^{(1)} \equiv \int_{-1}^{1} d x S\left(\sqrt{\left(k_{n}^{2}+k_{m}^{2}-2 k_{n} k_{m} x\right)}\right) \\
I_{s}^{(2)} \equiv \int_{-1}^{1} d x x^{2} S\left(\sqrt{\left(k_{n}^{2}+k_{m}^{2}-2 k_{n} k_{m} x\right)}\right)
\end{gathered}
$$

and $I_{A}$ and $I_{B}$ are the same as in reference [25],

$$
\begin{aligned}
I_{A}\left(l, k_{n}, k_{m}\right) & \equiv \int_{0}^{R_{M T}} d r y_{l+1}\left(r, k_{m}\right) \\
\times & \left(\frac{\partial y_{l_{i}}\left(r, k_{n}\right)}{\partial r}-(l+1) \frac{y_{l}\left(r, k_{n}\right)}{r}\right) \\
I_{B}\left(l, k_{n}, k_{m}\right) & \equiv \int_{0}^{R_{M T}} d r y_{l-1}\left(r, k_{m}\right) \\
& \times\left(\frac{\partial y_{l_{i}}\left(r, k_{n}\right)}{\partial r}+l \frac{y_{l}\left(r, k_{n}\right)}{r}\right)
\end{aligned}
$$


The sums over the orbital angular momentum quantum number $l$ appearing in equations (10) to (12) formally require an infinity of terms. Fortunately, in practice the sums converge quickly with a typical maximum $l$ being $\sim 15$. A larger number of partial waves is needed as the electrons become less degenerate. This point is further discussed in the context of the Ziman-Evans formula in reference $[17,18]$.

In the limit $S(k) \rightarrow 1 \forall k, \sigma^{(2)}(\omega)=0$ and the expression for the conductivity is reduced to that of Johnson's result $[22,25]$ provided that the integral over the muffin tin volume $V^{M T}=4 / 3 \pi R_{M T}^{3}$ is instead taken over all space. We return to this point in section IV

As shown in reference [25] the thermal conductivity $\kappa$ can be calculated in a straightforward extension. For a plasma of temperature $T$

$$
\kappa=\frac{1}{T}\left(\mathscr{L}_{22}-\frac{\mathscr{L}_{12}^{2}}{\mathscr{L}_{11}}\right)
$$

where (see also ref. [31])

$$
\begin{aligned}
\mathscr{L}_{n m}== & (-1)^{n+m} 2 \pi n_{I}^{0} \int d \epsilon\left(\epsilon-\mu_{e}\right)^{n+m-2} \\
& \times \frac{\partial f(\epsilon)}{\partial \epsilon} \int d \hat{\boldsymbol{k}}_{m} \int d \hat{\boldsymbol{k}}_{n} \\
& \times\left|\sqrt{k_{n} k_{m}} \bar{J}_{m n}\right|^{2} S\left(\left|\boldsymbol{k}_{n}-\boldsymbol{k}_{m}\right|\right)
\end{aligned}
$$

Clearly, $\sigma_{D C}=\sigma(0)=\mathscr{L}_{11}$. The Lorenz number is defined as

$$
L=\frac{\kappa}{T \sigma_{D C}}
$$

For fully degenerate electrons this takes the value $L=$ $\pi^{2} / 3$, while for fully non-degenerate electrons this takes the value 1.597 when electron-electron collisions are included. If electron-electron collision are not included then the non-degenerate limit becomes 4 [32, 33]. In the present KG formulation electron-electron collisions are included indirectly, since the wave functions are to be calculated in the presence of an electron exchange and correlation potential. Faussurier et al [34, 35] have recently developed a simple expression based on the KuboGreenwood formula (not including the ionic structure explicitly) that uses the Kramers formula. In that work they find a non-degenerate limit of 1 , presumably as a result of neglect of the Gaunt factor and a Drude renormalization procedure. Lastly we note that the extension of this formulation to mixtures is straightforward, but is not explored here.

\section{ZIMAN-EVANS EXPRESSION WITH AN EXPLICIT DENSITY OF STATES}

The Ziman-Evans expression for the inverse conductivity $R$ is $[30,36]$

$$
R=\frac{1}{\sigma_{D C}}=-\frac{1}{3 \pi^{2}\left(n_{e}^{*}\right)^{2}} \int_{0}^{\infty} d \epsilon \frac{d f}{d \epsilon} v^{3} \frac{1}{\tau_{p}}
$$

where $n_{e}^{*}$ is the density of scattering electrons and $f\left(\epsilon, \mu_{e}^{*}\right)$ is the Fermi-Dirac occupation factor and we have expanded the notation to indicate that it depends on the chemical potential $\mu_{e}^{*}$. The relaxation time $\tau_{p}$ is defined in terms of the generalized momentum transport cross section

$$
\sigma_{T R}(p)=2 \pi \int_{0}^{\pi} d \theta\left|T_{\boldsymbol{p p}^{\prime}}\right|^{2}(1-\cos \theta) \sin \theta
$$

and the transition matrix element $T_{\boldsymbol{p} \boldsymbol{p}^{\prime}}$ is given by [30]

$$
\left|T_{\boldsymbol{p} \boldsymbol{p}^{\prime}}\right|^{2}=\frac{d \sigma}{d \theta}(p, \theta) S(q)
$$

where $q^{2}=2 p^{2}(1-\cos \theta)$, and $S(q)$ is the ion-ion structure factor and $d \sigma / d \theta$ is the differential scattering cross section [14]. $\boldsymbol{p}\left(\boldsymbol{p}^{\prime}\right)$ is the initial (final) momentum of the electron before (after) the collision with the atom. Only elastic collisions are included, in which $|\boldsymbol{p}|=\left|\boldsymbol{p}^{\prime}\right|$. The final result for the relaxation time is

$$
\frac{1}{\tau_{p}}=\pi n_{I}^{0} \frac{v}{p^{4}} \int_{0}^{2 p} d q q^{3} \frac{d \sigma}{d \theta}(p, \theta) S(q)
$$

where $p=m_{e} v=\sqrt{2 \epsilon}$.

As explained in reference [14] a challenge when using equation (20) is the ambiguity in what one should choose for $n_{e}^{*}$ and $\mu_{e}^{*}$. This is due to the implicit free-electron density of states. Equation (20) is modified to include a non-free electron density of states by introducing a factor $N_{e}(\epsilon)$ into the integrand [37]:

$$
R=\frac{1}{\left(n_{e}^{*}\right)^{2}} \int_{0}^{\infty} d \epsilon\left(-\frac{d f}{d \epsilon}\right) N_{e}(\epsilon) \frac{1}{\tau_{p}}
$$

where

$$
N_{e}(\epsilon)=n_{I}^{0} \int_{0}^{\epsilon} d \epsilon^{\prime} \chi\left(\epsilon^{\prime}\right)
$$

$\chi(\epsilon)$ is the density of states such that the number of valence electrons per atom $\bar{Z}$ is

$$
\bar{Z}=\frac{\bar{n}_{e}^{0}}{n_{I}^{0}}=\int_{0}^{\infty} d \epsilon \chi(\epsilon) f\left(\epsilon, \mu_{e}\right)
$$

Here $\mu_{e}$ is the calculated, physical chemical potential and $\bar{n}_{e}^{0}$ is the density of valence electrons. Thus we can identify $n_{e}^{*}=\bar{n}_{e}^{0}$ and $\mu_{e}^{*}=\mu_{e}$. Such a choice cannot be used with equation (20) without introducing an inconsistency, and one is forced to compromise (see [14]). In the limit as $T \rightarrow 0$ in equation (24) we recover the expected Drude form

$$
\frac{1}{R}=\bar{n}_{e}^{0} \tau_{v_{F}}
$$

The usual expression for the resistivity (20) is recovered from equation (24) if we take the density of states to be its free electron form

$$
\chi^{\text {free }}(\epsilon)=\frac{\sqrt{2 \epsilon}}{n_{I}^{0} \pi^{2}}
$$


then

$$
N_{e}^{\text {free }}(\epsilon)=\frac{v^{3}}{3 \pi^{2}}
$$

\section{CONNECTION TO THE PAMD MODEL}

The above formulations can be used with any model that give access to the electron scattering potential $V^{\text {scatt }}$ and the ion-ion structure factor $S(k)$. From the scattering potential one can determine the chemical potential $\mu_{e}$, the wavefunctions $y_{l}(r, k)$, and the density of states $\chi(\epsilon)$. As in reference [14] we shall use the pseudoatom molecular dynamics (PAMD) [28] model to generate these inputs. This model has been described in detail elsewhere $[28,29,38]$ and has successfully been used to calculate equation of state, ionic structure and ionic transport [39] in the warm and hot dense matter regime. Its connection with the $\mathrm{ZE}$ equations was explored in detail in [14]. In short, in PAMD the electronic structure of one pseudo-atom in a plasma is calculated using density functional theory (here we restrict ourselves to Kohn-Sham DFT ${ }^{1}$ ). By coupling this electronic structure to the integral equations of fluid theory (the quantum Ornstein-Zernike (QOZ) equations) one calculates a parameter-free ion-ion pair interaction potential. This can be used in molecular dynamics simulations or, as we have here and in reference [14], directly in the QOZ equations to generate $S(k)$.

As discussed in reference [14] there are at least two reasonable choices for the scattering potential: the pseudoatom potential $V^{P A}(r)$ and the average atom potential $V^{A A}(r)$. The former can be used to construct the total scattering potential of the plasma when combined with a set of nuclear position vectors $\left\{\boldsymbol{R}_{i}\right\}$

$$
V^{t o t}(\boldsymbol{r})=\sum_{i} V^{P A}\left(\left|\boldsymbol{r}-\boldsymbol{R}_{i}\right|\right)
$$

while the latter $V^{A A}$ is a muffin-tin like potential that extends to the ion-sphere radius. Given the above derivation of the KG formula one would expect $V^{A A}$ to be the most reasonable choice because it the derivation assumes a muffin-tin form of the potential. However, it was found in [14] that when using the (similarly derived) ZE formulation $V^{P A}$ gives generally much better agreement with KS-DFT-MD results that are thought to be accurate even though $V^{P A}$ is not of muffin-tin form (unless one admits a very large overlap of the muffin-tin spheres). This surprising result was explained by realizing that in the Born limit of scattering (i.e. where the scattered state is a plane wave) the total scattering cross section

\footnotetext{
${ }^{1}$ For the results in section $\mathrm{V}$ we have used the zero temperature Local Density Approximation [40] for aluminum and the finite temperature LDA for beryllium and hydrogen [41].
}

separates into a sum over cross sections for each atom. In that limit the total scattering cross section is given by the Fourier transform of $V^{\text {tot }}$. Thus to recover the Born limit it is necessary to use the $V^{P A}$ potential. Though this choice violates the condition of non-overlapping muffintins, it compensates for the neglect of multiple scattering effects in our wavefunctions by essentially treating them in the Born approximation, while allowing strong single site scattering though the use of the t-matrix approximation.

In the KG formulation we similarly neglect multiple scattering contributions and we might expect the same potential $\left(V^{P A}\right)$ to also lead to improved results, again letting the muffin tin volume being replaced by a integral over all space. We note that in Johnson's formulation [22], where $S(k)=1$, the $V^{A A}$ potential is used and the integral is taken over all space. There, the physical model is an average atom model in jellium. One could restrict the integrals to be inside the muffin-tin (ion-sphere) volume only, but this results in conductivities that are orders of magnitude incorrect! The reason for this gross error was first suggested in reference [42]. In that work the isolated cluster method (ICM) was used to calculate conductivity of clusters of atoms with up to 201 atoms using the real-space Green's function method. The average atom model is essentially an ICM with one atom [43]. In reference [42] they found that the conductivity was dependent on the size of the cluster and that convergence was not achieved with up to 201 atoms. The explanation given was that the cluster approach can only yield correct results if the mean free path of the electron is approximately equal to, or smaller than, the size of the cluster - if the mean free path of the electron is larger than the the cluster, the scattering processes that lead to a finite conductivity cannot be expected to be accurately modeled in the cluster representation [42]. Clearly, here with our neglect of multiple scattering effects, our cluster size is effectively the volume of the ion-sphere, hence unless the resistivity is very large (corresponding to very small mean free paths) the results will be substantially incorrect. By letting the integration volume go to infinity this obviously corrects for this defect. However the price is that, since no other scattering events are included in the electronic wavefunction, the calculated conductivity diverges as $\omega^{-2}$ [22]. Johnson introduced a practical method to correct for this divergence: the optical conductivity is required to have a Drude like behavior by ad hoc multiplying by $\omega^{2} /\left(\gamma^{2}+\omega^{2}\right)$. $\gamma$ is found by enforcing the frequency sum rule

$$
\int_{0}^{\infty} d \omega \sigma(\omega)=\frac{n_{I}^{0} \pi \bar{Z}}{2}
$$

where $\bar{Z}$ is the number of valence electrons per ion. We note that we are only considering the free-free contribution to the conductivity here. This combination of letting the integration volume go to infinity and enforcing the Drude form represents a reasonable way of captur- 


\begin{tabular}{ll}
\hline \hline Notation & Description \\
\hline KG: $V^{P A}$ & Equation (9) with wave functions calculated in the $V^{P A}(r)$ potential. \\
KG: $V^{A A}$ & Equation (9) with wave functions calculated in the $V^{A A}(r)$ potential. \\
KG: $V^{A A}, S(k)=1$ & Same as KG: $V^{A A}$, but with $S(k)=1$. This is equivalent to Johnson's formulation [22]. \\
\hline ZE-DOS: $V^{A A}$ or $V^{P A}$ & Equation (24) with either potential. \\
ZE- $\mu_{e}$ & Equation (20) with the physical chemical potential (see [14]). \\
ZE- $\bar{\mu}_{e}$ & Equation (20) with the free electron chemical potential (see [14]). \\
\hline \hline
\end{tabular}

TABLE I: Descriptions of notation used in the figures.

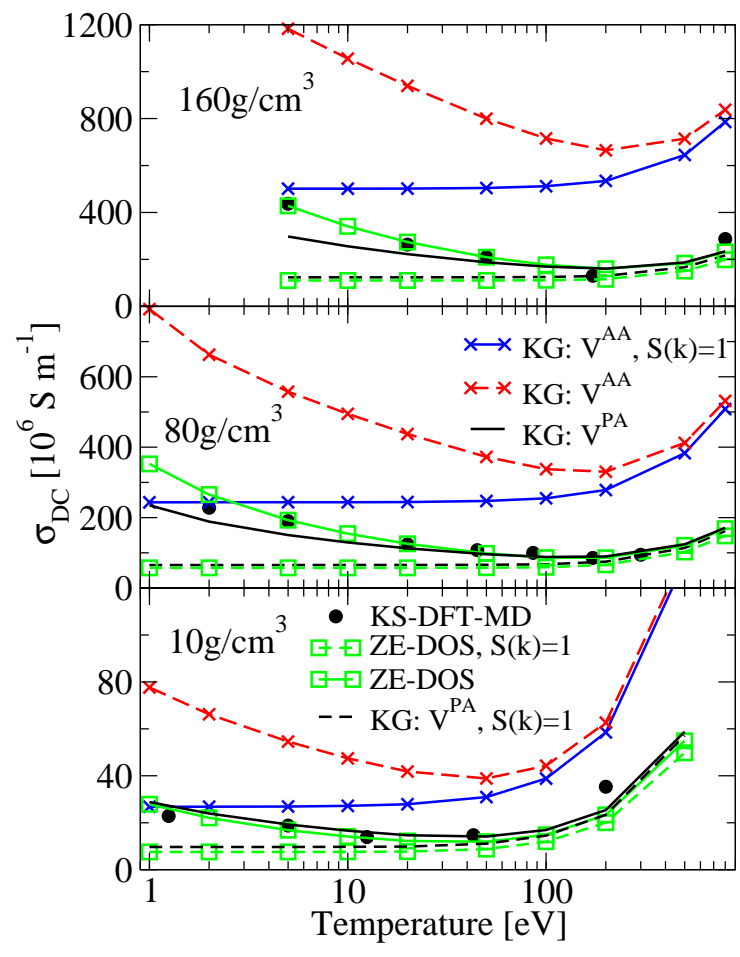

FIG. 1: (Color online) Electrical conductivity of dense hydrogen. The KS-DFT-MD results are from reference [1].

ing the main effects important to the calculation of the conductivity, but clearly introduces a significant source of uncertainty into the quality of the calculation.

In the next section we present calculations using the KG equation (9) using this infinite volume correction. We show results using the two potentials $V^{P A}$ and $V^{A A}$. For $V^{A A}$ we also show the result with $S(k)=1$, which is the same as Johnson's formulation [22], these can be compared to the nearly identical calculations presented in reference [25]. We also show results from the ZimanEvans equation with the non-free density of states included, equation (24), that we label ZE-DOS. Our notation is summarized in table I.

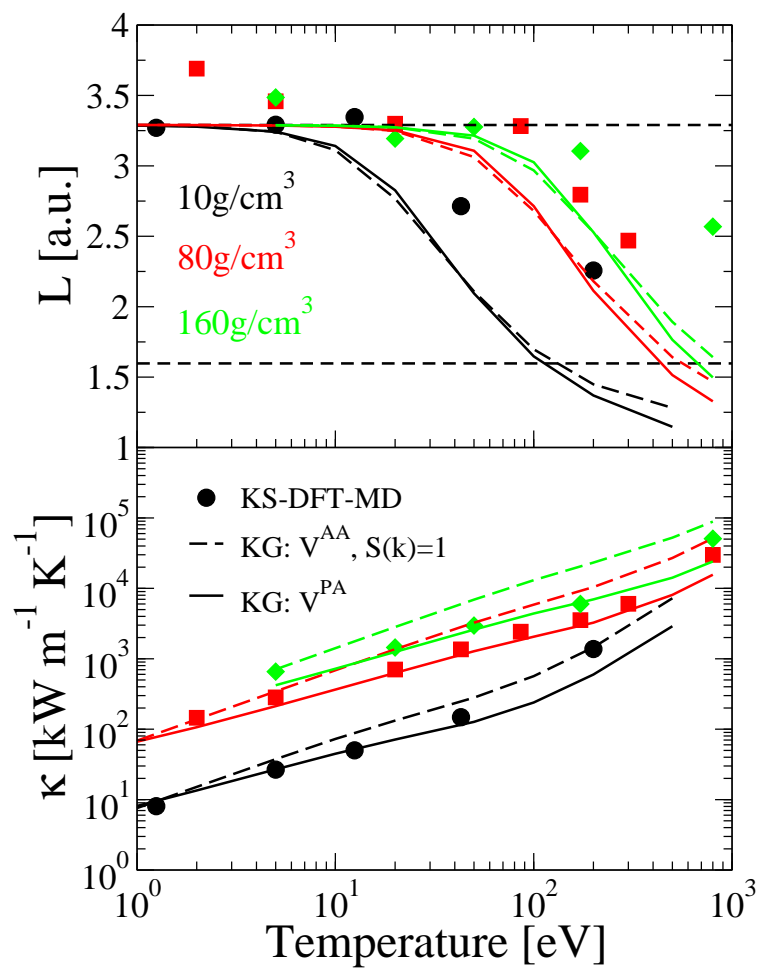

FIG. 2: (Color online) Lorenz number and thermal conductivity of dense hydrogen. The KS-DFT-MD results are from reference [1]. The horizontal dashed lines for the Lorenz number indicate the degenerate and non-degenerate limits.

\section{RESULTS}

In figure 1 we show results for the DC conductivity $\sigma_{D C}$ for dense hydrogen. We compare to KS-DFTMD results from [1]. Clearly both the Ziman-Evans (ZE-DOS) calculations based on equation (24) and the Kubo-Greenwood (KG) calculations based on equation (9) agree well with the KS-DFT-MD results when the pseudoatom potential $\left(V^{P A}\right)$ is used. A comparison of the ZE-DOS results to those presented in reference [14] with the two choices of chemical potential (not shown, but compare to results in reference [14]) reveals little effect from the new formulation, as we would expect, since the two results in [14] are similar. Where a difference 


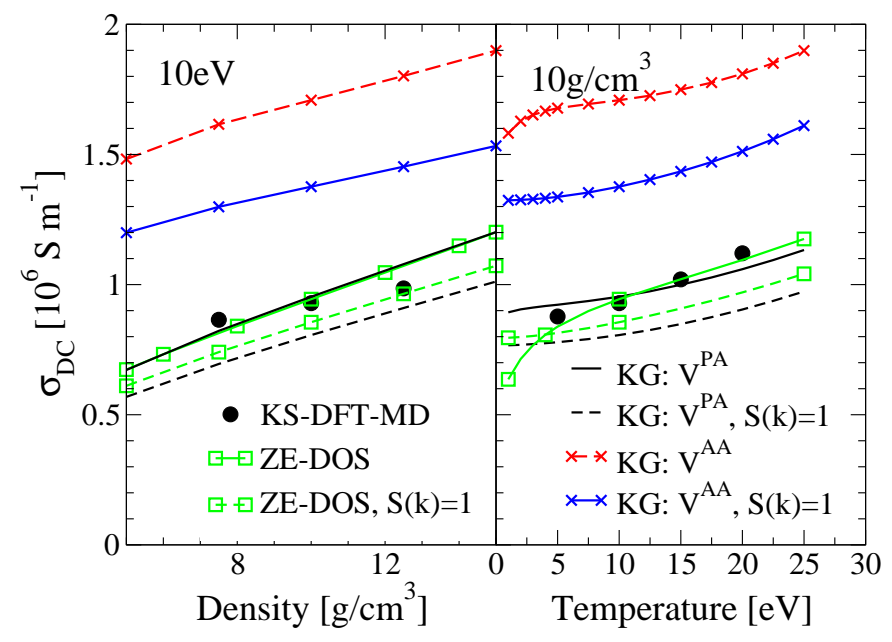

FIG. 3: (Color online) Electrical conductivity of dense beryllium. The KS-DFT-MD results are from references $[5,25]$.

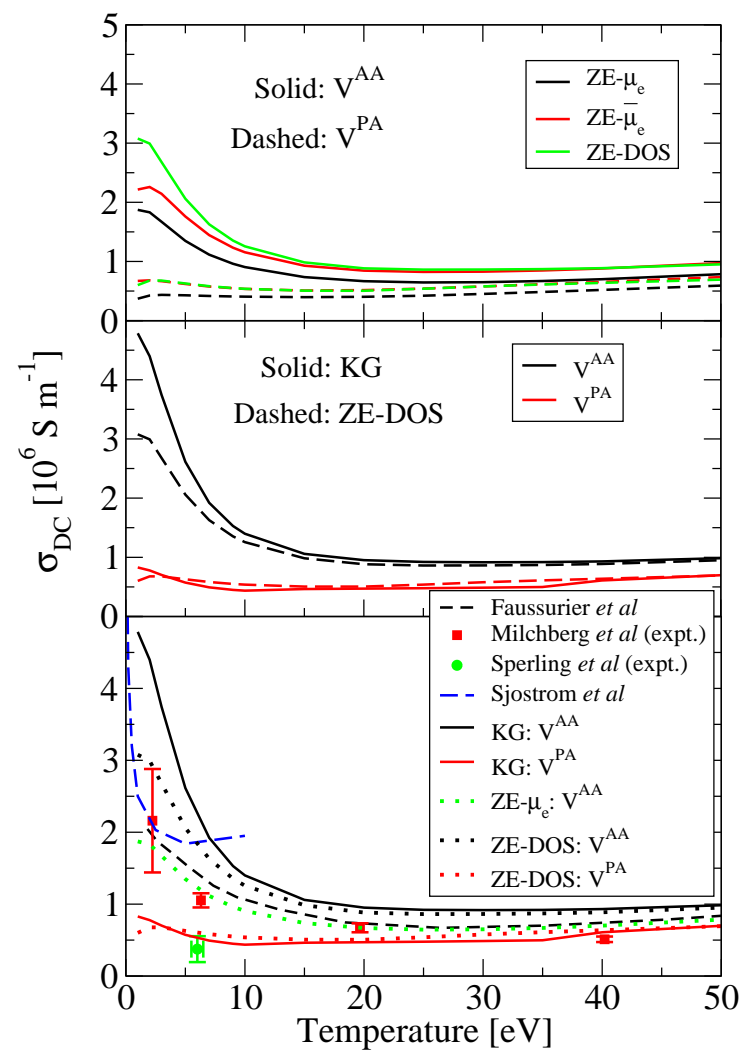

FIG. 4: (Color online) Electrical conductivity of solid density $\left(2.7 \mathrm{~g} / \mathrm{cm}^{3}\right)$ aluminum.

is seen the ZE-DOS calculations most closely follows the calculations based on the choice $\bar{\mu}_{e}$. KG calculations using $V^{A A}$ are also shown in figure 1 . When the structure factor is included the qualitative agreement is reasonable but the quantitative agreement is poor. Without the structure factor, even the qualitative agreement is

\begin{tabular}{ccc}
\hline \hline & $10 \mathrm{kK}$ & $30 \mathrm{kK}$ \\
KS-DFT-MD [6] & 1.70 & 1.25 \\
ZE-DOS: $V^{A A}$ & 2.19 & 1.82 \\
ZE-DOS: $V^{A A}, S(k)=1$ & 1.18 & 1.22 \\
ZE- $\bar{\mu}_{e}: V^{A A}$ & 1.12 & 1.30 \\
ZE-DOS: $V^{P A}$ & 0.67 & 0.65 \\
ZE-DOS: $V^{P A}, S(k)=1$ & 0.45 & 0.47 \\
KG: $V^{A A}$ & 3.28 & 2.59 \\
KG: $V^{A A}, S(k)=1$ & 1.93 & 1.86 \\
$\mathrm{KG}: V^{P A}$ & 0.72 & 0.64 \\
$\mathrm{KG}: V^{P A}, S(k)=1$ & 0.41 & 0.44 \\
\hline \hline
\end{tabular}

TABLE II: A comparison of the predictions of the DC conductivity in units of $10^{6} \mathrm{~S} \mathrm{~m}^{-1}$ from the present models to the calculations of Desjarlais et al [6] for aluminum at $2 \mathrm{~g} / \mathrm{cm}^{3}$ at two temperatures (10 and $30 \mathrm{kK}$ ).

poor and the results agree with those presented in [25]. Therefore the combination of the use of $V^{P A}$ with the inclusion of the ionic structure factor are both vital to find agreement with the KS-DFT-MD results in this case.

In figure 2 we show calculations of the thermal conductivity $\kappa$ and Lorenz number $L$ for the same dense hydrogen conditions as in figure 1 . We show calculations using the average atom potential $V^{A A}$ with $S(k)=1$, and using $V^{P A}$. The results using $V^{A A}$ amount to essentially the same calculations as in [25], and the results are very similar, i.e. generally the thermal conductivity overestimates the KS-DFT-MD and the trends are only broadly in agreement. In contrast, the results using $V^{P A}$ agree well with KS-DFT-MD, though some differences at high temperature are found. For the Lorenz number using either potential leads to similar results, and both agree with the degenerate limit at low temperature, and tend to underestimate KS-DFT-MD at high temperature. Moreover, as was found in reference [25] the method does not recover the non-degenerate limit at the highest temperatures seen. This is likely to be related to the Drude renormalization (as in reference [34]), thought the exact cause remains unclear.

In figure 3 we show results for warm dense beryllium. As for hydrogen, using the $V^{A A}$ leads to an overestimation of the KS-DFT-MD results, and using $V^{P A}$ with $S(k)$ from PAMD leads to very good agreement, using either the ZE-DOS or the KG methods. These results, figures 1 to 3 , demonstrate that both models introduced in this work can successfully predict conductivities in warm dense matter. For clarity of the figures, we have not shown an explicit comparison for ZE-DOS to the results presented in [14] which used the original ZE equation (20) with the two choices of chemical potential (ZE- $\mu_{e}$ and $\left.\mathrm{ZE}-\bar{\mu}_{e}\right)$. However, a comparison of the results reveals that ZE-DOS generally lies between the two and closer to ZE- $\bar{\mu}_{e}$ than ZE- $\mu_{e}$.

In figure 4 we show results for warm dense aluminum. 
Here the situation is much more complicated. In the top panel we show calculations based on the Ziman-Evans formula only. We compare both potentials $\left(V^{A A}\right.$ and $V^{P A}$ ) as well as the new ZE-DOS formulation to original ZE formulation with the two choices of chemical potential considered in [14]. We find that the results from ZE-DOS agree reasonably closely with $\mathrm{ZE}-\bar{\mu}_{e}$ and the the results using $V^{A A}$ are larger than using $V^{P A}$. In the center panel we compare the ZE-DOS results to those using the KG formulation. For the same potential the $\mathrm{ZE}$ and $\mathrm{KG}$ are reasonably close, but at lowest temperatures KG with $V^{A A}$ gives a somewhat larger conductivity than $\mathrm{ZE}$ with the same potential.

In the bottom panel of figure 4 we compare to other calculations and experiments. First there is an experiment due to Milchberg et al [26] and a very recent experiment due to Sperling et al [27]. The experiments are in significant disagreement with each other. A recent simulation that post-processes an Orbital Free MD simulation with the Kohn-Sham KG method is also shown (Sjostrom et al $[7])$. In the analysis of reference [7] they demonstrate that their simulations agree with previous KS-DFT-MD results at lower temperatures as well as other experiments, casting doubt on the veracity of the new measurements of Sperling et al. Finally we also show a calculation of Faussurier et al that uses the SCAALP model [15]. This is a model that is similar in spirit to the PAMD model used here, though differs in details. Their conductivity calculation uses the Ziman-Evans method and is qualitatively most similar to our calculations when we use ZE- $\mu_{e}$ with $V^{A A}$. Comparing the result of Faussurier et al to this calculation (bottom panel) we find good agreement which provides gross check that our calculations are reasonable.

Now comparing our calculations using $V^{P A}$ with ZEDOS or KG to the simulations of Sjostrom et al we find that while they agree well with each other, they do not agree well the results of Sjostrom et al [7], with our calculations predicting a significantly smaller conductivity. Moreover our results are in agreement with the recent experiment of Sperling et al but underestimate the older experiment of Milchberg et al. Our results using $V^{A A}$ with either ZE-DOS or KG also do not agree well with the results of Sjostrom et al either, though the magnitude is improved. In fact the best agreement with the Sjostrom simulations is found when we emulate the calculation of Faussurier et al (i.e. ZE- $\mu_{e}: V^{A A}$ ). The evidence put forward in Sjostrom et al [7] suggests that the experiment of Sperling, and in turn our results using $V^{P A}$, are too small. Similar trends are seen in table II where we compare to a KS-DFT-MD calculation of Desjarlias et al [6] for aluminum at $2.0 \mathrm{~g} / \mathrm{cm}^{3}$ and at two temperatures. This significant disagreement between these simulations and our $V^{P A}$ results for aluminum stands in stark contrast to the excellent agreement found for hydrogen and beryllium (figures 1 and 3 ).

To explain why we find such a strong disagreement in this case we recall the reasoning behind the choice of the electron scattering potential $V^{P A}$. In the limit where the Born approximation is valid (i.e. when the scattered electron can be modeled as a plane wave) the total scattering cross section for the plasma separates into individual scattering cross sections for each ion in the plasma, which depend only on the scattering potential at that site. In the language of the PAMD model this scattering potential is $V^{P A}(r)$. Thus, the obvious approximation when ignoring multiple scattering effects, but wishing to allow strong scattering (i.e. beyond the Born approximation) is to continue to assume that each site scatters the electrons independently, but treat the scattering cross section using the t-matrix approach. In the KG formula the t-matrix is equivalent to using the calculated scattered wavefunctions. Thus we can expect $V^{P A}$ to be a reasonable scattering potential where ever multiple scattering effects can be ignored. One test that would indicate the validity of this assumption is if the Born calculation itself gives reasonable results. In fact, as shown in [14, 44], Born approximation based calculations using the ZE formula give reasonable results for the hydrogen and Beryllium cases we have looked at here, whereas for aluminum the Born result is in gross error. This is thus the likely reason for the failure of the $V^{P A}$ based calculation for aluminum at the lower temperatures. We stress that it is not a necessary condition that the Born approximation be valid for the present models to be accurate, rather that the multiple scattering effect be negligible. Indeed the t-matrix based calculations presented in [14] are in significantly better agreement with the KS-DFT-MD result for hydrogen than corresponding Born approximation results.

As shown in figure 4 , using $V^{A A}$ we get somewhat improved agreement with the simulations of Sjostrom et al. Unlike $V^{P A}, V^{A A}$ does not recover the Born limit (leading to the poor results using this potential in figures 1 and 3 ). Using $V^{A A}$ may partially compensate for the breakdown of the weak multiple scattering effect assumption, but not in a controlled way. Therefore it is difficult to know for which cases (i.e. element, densities and temperatures) that using $V^{A A}$ will lead to improved agreement. In light of this we must be cautious in interpreting this somewhat improved agreement as evidence that $V^{A A}$ leads to improved agreement in general at low temperature.

\section{CONCLUSIONS}

A new derivation of the Kubo-Greenwood conductivity for average atom models is given. The derivation improves upon the previous derivation of Johnson et al [22] by taking the ionic structure factor explicitly into account. Calculations based on the new expression, using the pseudoatom molecular dynamics model [28] to provide the inputs, result in a significant improvement in agreement with KS-DFT-MD simulations for the hydrogen and beryllium plasmas tested. We have also given 
and tested a version of the Ziman-Evans formula for the resistivity that takes into account a non-free electron density of states. This new expression removes the ambiguity as to the choice of chemical potential and density of scattering electrons that was discussed in details in [14]. The new Ziman-Evans formula also gives good agreement with the KS-DFT-MD results for hydrogen and beryllium. We have also given the expression for the thermal conductivity based on the Kubo-Greenwood formulation, and found much improved agreement with KS-DFT-MD results for dense hydrogen.

Calculations from the new Ziman-Evans and KuboGreenwood formulas for warm dense aluminum found relatively poor agreement with ab initio simulations. Possible reasons for this are discussed.

\section{Acknowledgments}

This work was performed under the auspices of the United States Department of Energy under contract DEAC52-06NA25396 and LDRD number 20150656ECR.

\section{Appendix A: Aspects of the derivation of the KG formula}

When deriving equation (9) we are faced with an integral of the form

$$
\begin{aligned}
I \equiv & \int d \hat{\boldsymbol{k}} \int d \hat{\boldsymbol{k}}^{\prime} S\left(\left|\boldsymbol{k}-\boldsymbol{k}^{\prime}\right|\right) \\
& \times Y_{l_{1}, m_{1}}(\hat{\boldsymbol{k}}) Y_{l_{2}, m_{2}}^{*}\left(\hat{\boldsymbol{k}}^{\prime}\right) Y_{l_{3}, m_{3}}^{*}(\hat{\boldsymbol{k}}) Y_{l_{4}, m_{4}}\left(\hat{\boldsymbol{k}}^{\prime}\right)(\mathrm{A} 1)
\end{aligned}
$$

[1] Flavien Lambert, Vanina Recoules, Alain Decoster, Jean Clerouin, and Michael Desjarlais. On the transport coefficients of hydrogen in the inertial confinement fusion regime a). Physics of Plasmas (1994-present), 18(5):056306, 2011.

[2] S. X. Hu, L. A. Collins, T. R. Boehly, J. D. Kress, V. N. Goncharov, and S. Skupsky. First-principles thermal conductivity of warm-dense deuterium plasmas for inertial confinement fusion applications. Phys. Rev. E, 89:043105, Apr 2014.

[3] Ryogo Kubo. Statistical-mechanical theory of irreversible processes. i. general theory and simple applications to magnetic and conduction problems. Journal of the Physical Society of Japan, 12(6):570-586, 1957.

[4] D.A. Greenwood. The boltzmann equation in the theory of electrical conduction in metals. Proceedings of the Physical Society, 71(4):585, 1958.

[5] David E. Hanson, Lee A. Collins, Joel D. Kress, and Michael P. Desjarlais. Calculations of the thermal conductivity of national ignition facility target materials at temperatures near $10 \mathrm{ev}$ and densities near $10 \mathrm{~g} / \mathrm{cc}$ using finite-temperature quantum molecular dynamics. Physics of Plasmas, 18(8), 2011.

[6] M. P. Desjarlais, J. D. Kress, and L. A. Collins. Electri-

$$
\sum_{m=-l}^{l} m^{2}\left|Y_{l m}(\hat{\boldsymbol{k}})\right|^{2}=\frac{l(l+1)(2 l+1)}{8 \pi} \sin ^{2} \theta_{k}
$$

which simplifies to

$$
I=\underset{\quad}{\int d \hat{\boldsymbol{k}} S(k) Y_{l_{1}, m_{1}}(\hat{\boldsymbol{k}}) Y_{l_{3}, m_{1}}^{*}(\hat{\boldsymbol{k}})}
$$

where $\delta$ is the Kronecker delta, and $k=$ $\sqrt{k^{2}+k^{\prime 2}-k k^{\prime} \cos \theta_{k}}$. When $S(k)=1$, the integrals in $I$ separate and the result simplifies to $\delta_{l_{2}, l_{4}} \delta_{m_{2}, m_{4}} \delta_{l_{1}, l_{3}} \delta_{m_{1}, m_{3}}$. To eliminate the remaining sum of magnetic quantum number $m$ a useful relation is

cal conductivity for warm, dense aluminum plasmas and liquids. Phys. Rev. E, 66:025401, Aug 2002.

[7] Travis Sjostrom and Jérôme Daligault. Ionic and electronic transport properties in dense plasmas by orbitalfree density functional theory. Phys. Rev. E, 92:063304, 2015.

[8] R. P. Feynman, N. Metropolis, and E. Teller. Equations of state of elements based on the generalized fermithomas theory. Phys. Rev., 75:1561-1573, May 1949.

[9] David A. Liberman. Self-consistent field model for condensed matter. Phys. Rev. B, 20:4981-4989, Dec 1979.

[10] R. Piron and T. Blenski. Variational-average-atomin-quantum-plasmas (vaaqp) code and virial theorem: Equation-of-state and shock-hugoniot calculations for warm dense al, fe, cu, and pb. Phys. Rev. E, 83:026403, Feb 2011.

[11] B. Wilson, V. Sonnad, P. Sterne, and W. Isaacs. Purgatorioa new implementation of the inferno algorithm. Journal of Quantitative Spectroscopy and Radiative Transfer, 99(13):658 - 679, 2006. Radiative Properties of Hot Dense Matter.

[12] Gérald Faussurier, Christophe Blancard, Philippe Cossé, and Patrick Renaudin. Equation of state, transport coefficients, and stopping power of dense plasmas from the 
average-atom model self-consistent approach for astrophysical and laboratory plasmas. Physics of Plasmas, 17(5), 2010.

[13] Balazs F. Rozsnyai. Relativistic hartree-fock-slater calculations for arbitrary temperature and matter density. Phys. Rev. A, 5:1137-1149, Mar 1972.

[14] D.J. Burrill, D.V. Feinblum, M.R.J. Charest, and C.E. Starrett. Comparison of electron transport calculations in warm dense matter using the ziman formula. High Energy Density Physics, 19:1 - 10, 2016.

[15] Gérald Faussurier and Christophe Blancard. Resistivity saturation in warm dense matter. Phys. Rev. E, 91:013105, Jan 2015.

[16] P.A. Sterne, S.B. Hansen, B.G. Wilson, and W.A. Isaacs. Equation of state, occupation probabilities and conductivities in the average atom purgatorio code. High Energy Density Physics, 3(12):278 - 282, 2007. Radiative Properties of Hot Dense Matter.

[17] François Perrot and M. W. C. Dharma-wardana. Electrical resistivity of hot dense plasmas. Phys. Rev. A, 36:238-246, Jul 1987.

[18] J.C. Pain and G. Dejonghe. Electrical resistivity in warm dense plasmas beyond the average-atom model. Contributions to Plasma Physics, 50(1):39-45, 2010.

[19] M. W. C. Dharma-wardana. Static and dynamic conductivity of warm dense matter within a density-functional approach: Application to aluminum and gold. Phys. Rev. E, 73:036401, Mar 2006.

[20] Balazs F. Rozsnyai. Electron scattering in hot/warm plasmas. High Energy Density Physics, 4(1):64-72, 2008.

[21] S. Mazevet, L.A. Collins, N.H. Magee, J.D. Kress, and J.J. Keady. Quantum molecular dynamics calculations of radiative opacities. Astronomy \& Astrophysics, 405(1):L5-L9, 2003.

[22] W.R. Johnson, C. Guet, and G.F. Bertsch. Optical properties of plasmas based on an average-atom model. Journal of Quantitative Spectroscopy and Radiative Transfer, 99(13):327 - 340, 2006. Radiative Properties of Hot Dense Matter.

[23] W.R. Johnson. Low-frequency conductivity in the average-atom approximation. High Energy Density Physics, 5(12):61 - 67, 2009.

[24] M. Yu. Kuchiev and W. R. Johnson. Low-frequency plasma conductivity in the average-atom approximation. Phys. Rev. E, 78:026401, Aug 2008.

[25] C. E. Starrett, J Clérouin, V Recoules, J. D. Kress, L. A. Collins, and D. E. Hanson. Average atom transport properties for pure and mixed species in the hot and warm dense matter regimes. Physics of Plasmas (1994present), 19(10):102709, 2012.

[26] H. M. Milchberg, R. R. Freeman, S. C. Davey, and R. M. More. Resistivity of a simple metal from room temperature to $10^{6}$ k. Phys. Rev. Lett., 61:2364-2367, Nov 1988.

[27] P. Sperling, E. J. Gamboa, H. J. Lee, H. K. Chung, E. Galtier, Y. Omarbakiyeva, H. Reinholz, G. Röpke, U. Zastrau, J. Hastings, L. B. Fletcher, and S. H. Glenzer. Free-electron x-ray laser measurements of collisionaldamped plasmons in isochorically heated warm dense matter. Phys. Rev. Lett., 115:115001, 2015.

[28] C. E. Starrett, J. Daligault, and D. Saumon. Pseudoatom molecular dynamics. Phys. Rev. E, 91:013104, Jan 2015.

[29] C. E. Starrett and D. Saumon. Electronic and ionic structures of warm and hot dense matter. Phys. Rev. E, 87:013104, Jan 2013.

[30] R. Evans, B. L. Gyorfey, N. Szabo, and J. M. Ziman. On the resistivity of liquid transition metals. In S. Takeuchi, editor, The properties of liquid metals. Taylor and Francis, London, 1973.

[31] Bastian Holst, Martin French, and Ronald Redmer. Electronic transport coefficients from ab initio simulations and application to dense liquid hydrogen. Phys. Rev. B, 83:235120, Jun 2011.

[32] H. Reinholz, G. Röpke, S. Rosmej, and R. Redmer. Conductivity of warm dense matter including electronelectron collisions. Physical Review E, 91(4):043105, 2015.

[33] Heidi Reinholz, Ronald Redmer, and Stefan Nagel. Thermodynamic and transport properties of dense hydrogen plasmas. Phys. Rev. E, 52:5368-5386, Nov 1995.

[34] G. Faussurier, C. Blancard, P. Combis, and L. Videau. Electrical and thermal conductivities in dense plasmas. Physics of Plasmas (1994-present), 21(9):092706, 2014.

[35] G. Faussurier and C. Blancard. Asymptotic regimes for the electrical and thermal conductivities in dense plasmas. Physics of Plasmas, 22(4), 2015.

[36] J.M. Ziman. A theory of the electrical properties of liquid metals. i: The monovalent metals. Philosophical Magazine, 6(68):1013-1034, 1961.

[37] A. Yu Potekhin and D.G. Yakovlev. Electron conduction along quantizing magnetic fields in neutron star crusts. ii. practical formulae. Astronomy and Astrophysics, 314:341-352, 1996.

[38] C.E. Starrett and D. Saumon. A simple method for determining the ionic structure of warm dense matter. High Energy Density Physics, 10:35 - 42, 2014.

[39] Jérôme Daligault, Scott D. Baalrud, Charles E. Starrett, Didier Saumon, and Travis Sjostrom. Ionic transport coefficients of dense plasmas without molecular dynamics. Phys. Rev. Lett., 116:075002, 2016.

[40] Paul AM Dirac. Note on exchange phenomena in the thomas atom. Proceedings of the Cambridge Philosophical Society, 26:376, 1930.

[41] Valentin V. Karasiev, Travis Sjostrom, James Dufty, and S. B. Trickey. Accurate homogeneous electron gas exchange-correlation free energy for local spin-density calculations. Phys. Rev. Lett., 112:076403, Feb 2014.

[42] Lucian Dulca, John Banhart, and Gerd Czycholl. Electrical conductivity of finite metallic systems: Disorder. Phys. Rev. B, 61:16502-16513, 2000.

[43] C.E. Starrett. A green's function quantum average atom model. High Energy Density Physics, 16:18 - 22, 2015.

[44] D. Burrill, D. Feinblum, M. Charest, and C. Starrett. Modeling warm dense matter using quantum-mechanical density function theory. Los Alamos National Laboratory Computational Physics Workshop 2015 report, 2015. 